\title{
Reflets
}

Revue ontaroise d'intervention sociale et communautaire

\section{Perceptions de femmes cadres dans une entreprise typiquement masculine}

\section{Sika Eliev et Christiane Bernier}

Volume 9, numéro 2, automne 2003

Travail et mieux-être

URI : https://id.erudit.org/iderudit/011092ar

DOI : https://doi.org/10.7202/011092ar

Aller au sommaire du numéro

Éditeur(s)

Reflets : Revue ontaroise d'intervention sociale et communautaire

ISSN

1203-4576 (imprimé)

1712-8498 (numérique)

Découvrir la revue

Citer cet article

Eliev, S. \& Bernier, C. (2003). Perceptions de femmes cadres dans une entreprise typiquement masculine. Reflets, 9(2), 87-113.

https://doi.org/10.7202/011092ar

\section{Résumé de l'article}

Depuis une dizaine d'années, plusieurs recherches ont porté sur l'articulation travail-famille avec l'objectif d'expliquer pourquoi un si petit nombre de femmes accèdent à des postes de cadre ou de gestionnaire dans les entreprises. D'autres études se sont penchées sur le rapport entre l'organisation, la culture de l'entreprise comme telle et le fait d'être une femme en poste de responsabilité. Elles arguent qu'une culture typiquement masculine dans l'entreprise est un obstacle de taille à l'avancement des femmes dans la hiérarchie organisationnelle: c'est la fameuse thèse du "plafond de verre" développée depuis les années quatre-vingt. La présente recherche montre la perception que les femmes cadres ont de leur milieu de travail et d'elles-mêmes, lorsqu'elles occupent un poste de responsabilité dans des entreprises typiquement masculines du secteur industriel.
Tous droits réservés (C) Reflets : Revue ontaroise d'intervention sociale et communautaire, 2002
Ce document est protégé par la loi sur le droit d'auteur. L'utilisation des services d'Érudit (y compris la reproduction) est assujettie à sa politique d'utilisation que vous pouvez consulter en ligne.

https://apropos.erudit.org/fr/usagers/politique-dutilisation/ 


\title{
Perceptions de femmes cadres dans une entreprise typiquement masculine ${ }^{1}$
}

\author{
Sika Eliev \\ Étudiante, doctorat en sociologie, Université du Québec à Montréal \\ Christiane Bernier \\ Professeure, département de sociologie, Université Laurentienne
}

\section{Introduction}

Depuis une dizaine d'années, plusieurs recherches ont porté sur l'articulation travail-famille avec l'objectif d'expliquer pourquoi un si petit nombre de femmes accèdent à des postes de cadre ou de gestionnaire dans les entreprises. D'autres études se sont penchées sur le rapport entre l'organisation, la culture de l'entreprise comme telle et le fait d'être une femme en poste de responsabilité. Elles arguent qu'une culture typiquement masculine dans l'entreprise est un obstacle de taille à l'avancement des femmes dans la hiérarchie organisationnelle: c'est la fameuse thèse du «plafond de verre» développée depuis les années quatre-vingt. La présente recherche veut montrer quelle perception les femmes cadres ont de leur milieu de travail et d'elles-mêmes, lorsqu'elles occupent un poste de responsabilité dans des entreprises typiquement masculines du secteur industriel. Nous pensons ici à l'industrie minière, où les hommes sont dominants, à la fois parce qu'ils sont en majorité dans l'entreprise et parce qu'ils y occupent la plupart des postes de gestion et tous les postes de haute direction. 
"L'enquête avait pour premier objectif de découvrir, à partir des trajectoires de quelques-unes de ces femmes, les facteurs qui, selon elles, ont favorisé ou, au contraire, entravé leur carrière, ..."
Ce sujet nous a particulièrement interpellées car nous savons que la présence des femmes s'est faite plus tangible dans l'industrie minière depuis une quinzaine d'années. Autrefois réservé strictement aux hommes éduqués, les postes de cadre et de cadre supérieur dans cette industrie sont désormais accessibles aux femmes puisqu'elles s'intéressent en plus grand nombre que par le passé aux sciences naturelles et au génie. À titre d'indicateur, on peut noter leur présence croissante dans les universités canadiennes de 1996 à 1999 au sein des programmes de doctorat à plein temps en génie et sciences appliquées ${ }^{2}$, où le rapport homme/femme passe de 13,8\% en 1996 à 16,2 \% en 1999. Plusieurs femmes ayant ce type de formation scientifique occupent aujourd'hui des postes de cadres intermédiaires et, certaines, des postes de cadres supérieures, dans les entreprises traditionnelles masculines du secteur minier. Nous nous sommes demandé comment ces femmes cadres vivent leur insertion dans ce milieu doublement masculin, c'est-à-dire à la fois dans une entreprise traditionnellement masculine, n'ayant, jusqu'aux années quatrevingt, embauché que des hommes ${ }^{3}$, et dans la hiérarchie organisationnelle, qui est encore massivement réservée aux hommes ${ }^{4}$.

L'enquête avait pour premier objectif de découvrir, à partir des trajectoires de quelques-unes de ces femmes, les facteurs qui, selon elles, ont favorisé ou, au contraire, entravé leur carrière, c'està-dire leur progression vers leur poste actuel. Nous avons voulu, en second lieu, savoir comment se passe réellement dans le quotidien, jour après jour, leur vécu, les entendre expliquer comment elles concilient les exigences travail-famille, comment elles font face à leur situation de minoritaire sexuelle dans l'entreprise, et voir si, selon elles, le rapport homme-femme y reste un perpétuel enjeu. Finalement, nous désirions nous faire une idée du potentiel de changement que représentent ces femmes dans leur entreprise au regard de l'avancée des femmes dans des postes de responsabilité traditionnellement définis comme masculins. 


\section{L'enquête}

L'échantillon sur les femmes cadres dans le secteur minier est une composante d'un plus vaste échantillon qui a été constitué dans le cadre d'une recherche ${ }^{5}$ qui visait une comparaison entre des femmes $(n=33)$ occupant un poste de responsabilité dans deux milieux professionnels différents. Le premier retenu, où l'ensemble du personnel est majoritairement féminin, est le milieu des caisses populaires. Le second retenu, à très forte majorité masculine, est celui de l'exploitation minière, celui-ci nous a semblé tout désigné. C'est ce groupe qui retiendra notre attention ici.

L'enquête s'est déroulée de janvier à avril 1997. Le sous échantillon présenté ici est composé de dix-huit femmes. Sur les douze qui possèdent un diplôme universitaire en sciences, huit sont ingénieures (génie chimique, métallurgique ou biophysique); quatre ont un baccalauréat en sciences naturelles (biochimie). En ce qui concerne les six autres, quatre ont suivi des formations spécifiques au sein de l'entreprise, l'une a un baccalauréat en droit et l'autre un baccalauréat en commerce ${ }^{6}$. Elles occupent toutes un poste de cadre intermédiaire ou de cadre supérieur dans deux exploitations minières du Nord de l'Ontario. Elles répondent à des caractéristiques diverses: la plupart sont mariées $(n=14)$, ont un diplôme universitaire $(n=14)$ et ont un ou plusieurs enfants à la maison $(n=13)$, six d'entre elles en ayant trois ${ }^{7}$. La moyenne d'âge se situe à 41 ans. Le titre de la fonction occupée varie grandement: supervisor $(\mathrm{n}=6)$, superintendent $(\mathrm{n}=4)$, manager ou foreman $(\mathrm{n}=4)$ et autres: industrial hygienist; coordinator; employer relations representatives; research engineer. La taille des groupes d'employés sous leur supervision varie de 300 personnes ou plus $(n=3)$ à 10 personnes ou moins $(n=11)$ et la moyenne d'années de service dans l'entreprise est de 13,5 pour l'ensemble de l'échantillon. Dans cette enquête, elles ont collaboré à une entrevue semi structurée à partir d'un protocole relativement élaboré. Celui-ci voulait cerner la façon dont elles perçoivent leur carrière, leur poste, leur entreprise, le type de relations que leurs collègues masculins entretiennent avec elles, ainsi que ce qu'elles représentent relativement à l'avancement des femmes dans des emplois non traditionnels. 


\section{Fondements théoriques}

"Deux perspectives théoriques s'avèrent pertinentes... les théories sur le pouvoir et celles sur la conciliation travailfamille et le "plafond de verre...»

"...l'exploitation économique du travail domestique se répercute sur le marché de l'emploi..."
Deux perspectives théoriques s'avèrent pertinentes lorsque l'on veut étudier l'insertion des femmes dans l'entreprise: l'analyse féministe, particulièrement les théories sur le pouvoir et celles sur la conciliation travail-famille et le "plafond de verre» et l'analyse sociologique des organisations, au sein de laquelle nous retenons l'approche du fonctionnalisme stratégique.

\section{La conciliation travail-famille}

Il y a déjà quelques décennies que le féminisme radical a développé sa théorie du pouvoir en mettant en lumière la construction patriarcale de la société, et ses avatars: la socialisation sexuée, la domination masculine et l'exploitation sexuelle des femmes (Delphy 1970, 1998; Guillaumin 1978; Mathieu 1989; Devreux 1985). Là où ce courant théorique a particulièrement contribué à la compréhension actuelle que nous avons du rapport qu'entretiennent les hommes et les femmes au marché du travail, c'est dans l'analyse de l'articulation économique du travail effectué dans les sphères domestique et publique et dans la mise à jour de sa répartition selon les sexes. D'une part, les femmes constituent le groupe sexué sur lequel repose encore majoritairement l'obligation de la maintenance domestique et le maternage (Statistique Canada 1995; Statistique Canada et Condition féminine Canada 1997). D'autre part, la division même du marché du travail selon le sexe a un impact considérable sur la discrimination de la main-d'oeuvre féminine (Redskin 1984; Gunderson et Riddel 1988; Bernier et Laflamme 2000, 2001). En effet, de nombreuses analyses féministes ont montré que l'exploitation économique du travail domestique se répercute sur le marché de l'emploi révélant que les femmes reproduisent dans leurs occupations rémunérées, fortement ghettoïsées, les tâches qu'elles effectuent au foyer (David 1986; Shelton 1992; Armstrong et Armstrong 1978, 1990, Descarries et Corbeil 1995). Ou encore, lorsque ce n'est pas le cas, que leurs obligations familiales nuisent réellement à leur carrière (Vandelac 1985; Shelton 1992; Descarries et Corbeil 1995; McFarlane et al. 2000). Cette inégalité dans la 
production du travail rémunéré et non rémunéré porte plusieurs femmes à refuser des postes de direction pour rester disponibles pour leur famille (Commission des sciences et de la technologie au service du développement 1996), ou encore, les incite à interrompre leur carrière pour cause de maternités, provoquant ainsi une discontinuité dans leur parcours professionnel, ce qui rend leur profil moins compétitif sur le marché (Barrère-Maurisson 1992; David 1986; Descarries et Corbeil 1995; Kempeneers 1992). Ainsi, en plus d'être des blocages structurels à la présence des femmes sur le marché du travail, les normes discriminatoires sousjacentes à la structure patriarcale du marché de l'emploi trouvent aussi écho dans une norme de socialisation intériorisée par les femmes elles-mêmes, les menant à une forme d'autocensure professionnelle (Conseil consultatif national des sciences et technologies 1993) rendant encore moins probable leur accession aux postes supérieurs. Selon certaines auteures, ces contraintes demeureront, selon toute vraisemblance, insurmontables, tant et aussi longtemps qu'une transformation de la culture et des normes qui régissent l'organisation du travail et de la vie familiale ne seront pas profondément modifiées afin de permettre un meilleur équilibre entre les deux (Descarries et Corbeil 1996; Luxton et Reiter 1997).

\section{Le «plafond de verre»}

Mais en plus de cette difficile articulation travail-famille et de la ghettoïsation des emplois selon le sexe, les femmes sont aussi confrontées au "plafond de verre» à mesure qu'elles grimpent dans la hiérarchie organisationnelle. De nombreuses recherches sur le plafonnement de carrière des femmes ont été réalisées, tant au Canada qu'aux États-Unis, tant dans le secteur privé que public. Plusieurs de ces recherches ont porté sur le plafonnement dans l'administration publique (Naff 1994; Lemire et Des Marais 1998) mais peu se sont penchées sur l'avancement des femmes dans les entreprises du secteur primaire où elles sont encore très peu présentes, surtout dans les postes de direction et de gestion ${ }^{8}$.

Les études sur le plafond de verre portent sur deux aspects: la nature des barrières qui limitent la progression des femmes et la 
"...quel que soit le niveau hiérarchique, la culture organisationnelle est, fondamentalement, une culture masculine..." perception des femmes elles-mêmes sur le traitement qui leur est réservé dans le milieu de travail (Lemire et Des Marais 1998). De façon générale, les recherches ont démontré que, au delà de la comparaison des qualifications liées directement à l'emploi (formation, ancienneté, etc), divers autres déterminants doivent être pris en considération pour expliquer la faible représentativité des femmes aux postes de gestion: l'absence de mentorat chez les femmes, la faiblesse de leurs réseaux informels, leur refus de mobilité et, une culture organisationnelle masculine.

\section{L'analyse organisationnelle et la culture d'entreprise}

Les facteurs qui ont été identifiés dans les recherches sur le plafond de verre seront aussi soulevés dans des analyses qui proviennent d'une autre approche théorique, celle du fonctionnalisme stratégique en sociologie des organisations. Selon Michel Crozier (1977), quelle que soit la place qu'occupe un acteur dans le système, il peut, jusqu'à un certain point, contourner, utiliser ou adapter les normes de l'entreprise d'une façon qui lui soit fonctionnelle, c'est-à-dire en fonction de ses propres objectifs. Dit autrement, à l'intérieur d'un cadre normatif, l'acteur dispose d'une marge de manœuvre qui rend possible l'utilisation de certaines stratégies qui lui sont propres dans la poursuite de ses intérêts.

À partir de cette approche, certaines recherches féministes en sociologie du travail ont abordé la question de la discrimination dans l'emploi. Les résultats de ces recherches font aussi valoir que quel que soit le niveau hiérarchique, la culture organisationnelle est, fondamentalement, une culture masculine, et que plus on monte dans les échelons de la hiérarchie, plus on est confronté au «old boys'club» (Field et Santucci 1983; Colwill 1987; Landry 1990). Poursuivant dans ce courant de pensée, les recherches ont tenté de voir quels types de stratégies les femmes utilisaient ou développaient pour face à cette culture masculine du pouvoir (Davis et al. 1995; Coderre et al. 1999).

Dans une enquête menée auprès de quarante et une femmes gestionnaires travaillant dans quatre organisations différentes, 
"...le contexte organisationnel exerce une influence importante sur le comportement des femmes..."
Micheline DesRosiers (1993) est parvenue à établir un rapport entre certains types de stratégies utilisées par les femmes, leur succès dans le monde de la gestion et le type d'entreprise dans lequel elles travaillent. Elle identifie, ainsi, quatre types de stratégies portant sur:

1) les formes motrices de l'action (équilibrage des forces);

2) les forces restrictives de l'action (résistance subtile);

3) une combinaison des deux premières (confrontation) et,

4) une dernière stratégie qui consiste à dépolitiser, à annihiler les rapports de force avec l'adversaire (conformisme).

Selon elle, les stratégies 1 et 2 sont les plus utilisées par les femmes, mais, elles sont fonction de deux variables: la distribution numérique des sexes aux postes de gestion (celle-ci peut être mixte ou à forte dominance masculine) et le type de culture organisationnelle déployée dans l'entreprise (selon l'auteure, il existe deux types: une culture de conflits (respect de l'autorité et des procédures formelles) et une culture de collaboration (initiatives individuelles et formalisme réduit). En combinant les deux variables dans l'explication de l'utilisation des stratégies par les femmes, DesRosiers émet à un constat intéressant: les femmes qui travaillent dans les organisations mixtes utilisent plus de moyens que les autres pour améliorer leur sort. Elles semblent devoir moins dépolitiser les interactions que leurs consœurs des organisations à dominance masculine.Ainsi, on peut voir ainsi que le contexte organisationnel exerce une influence importante sur le comportement des femmes:

La première caractéristique [distribution numérique] laisse supposer que les barrières structurelles amènent les fermmes à être un peu moins actives dans les jeux d'influence, tandis que la seconde [la culture organisationnelle] inciterait à utiliser des stratégies en accord avec les valeurs et traditions du milieu. L'effet des deux variables signifie que ce ne sont pas seulement les caractéristiques structurelles plus objectives des organisations qui agissent sur les comportements mais aussi leurs caractéristiques culturelles plus subjectives (DesRosiers 1993:9). 
"...les femmes, ont

une très grande visibilité et doivent travailler plus que les hommes pour continuellement démontrer leur compétence.»
Il s'agit donc d'une dynamique complexe qui implique plusieurs niveaux d'analyse. Cette perspective mène à penser que le rapport n'est pas automatique ou mécanique entre la présence des femmes dans une entreprise et leur mise à l'écart systématique des postes de pouvoir. Dit autrement: les femmes doivent s'employer à contourner le "plafond de verre». Comme le souligne l'auteure, les femmes doivent apprendre quand saisir les occasions et les exploiter. Son modèle explicatif dégage une toute première piste permettant de s'interroger sur les femmes comme «actrices dans l'organisation" et non plus uniquement comme victimes passives d'une discrimination historique, sans réaction possible ou sans action autre qu'extérieure à leur propre activité.

Une autre recherche, celle de Rosabeth Moss Kanter (1977), part de l'analyse de la répartition numérique des groupes dans une organisation pour expliquer le rapport entre le pouvoir institutionnel et la place des femmes dans des postes de gestionnaires. Selon l'auteure, en augmentant le nombre des membres de la minorité dans un groupe, les attitudes deviennent plus positives à leur égard et l'on peut ainsi jouer sur les rapports de force. Ce qu'il faut, selon elle, c'est atteindre une masse critique. Kanter a construit un modèle théorique dans lequel elle identifie quatre types de rapport au pouvoir dans les entreprises en fonction de la répartition du nombre. La distribution inégale des groupes produit un certain nombre d'obstacles qui agissent sur les systèmes de possibilités, les structures de pouvoir et les perceptions sociales à l'intérieur du milieu. Ainsi, par exemple, en tant qu'alibis, les femmes, ont une très grande visibilité et doivent travailler plus que les hommes pour continuellement démontrer leur compétence. En outre, le contraste entre dominées et dominants peut aussi avoir pour effet de rendre ceux-ci plus conscients de leur propre culture et, pour préserver cette culture, les hommes, trop fréquemment, excluront leurs collègues féminines des réseaux informels et assimileront les rôles des femmes cadres aux rôles stéréotypés traditionnellement féminins.

Cette absence d'ouverture inciterait les femmes à adopter des attitudes peu propices à leur avancement, comme la perte d'intérêt pour les postes de responsabilité. De plus, ne pouvant développer 
l'échange intergroupe, à cause de ces blocages institutionnels ou par défaut de nombre, elles ne disposent pas d'appui organisationnel, ce qui ne leur facilite pas la tâche. En résumé, le modèle théorique de Kanter laisse présumer qu'une plus grande présence des femmes aux postes de direction changerait la dynamique culturelle dans les entreprises.

À l'aide de ces quelques éléments théoriques, il est possible de cerner les perceptions des femmes cadres interviewées. Quel type de trajectoire ont-elles eue? Que pense-t-elle de l'importance du nombre de femmes dans leur entreprise? Quels types de rapports existent-ils avec leurs collègues masculins? Quelle perception ontelle de leur articulation travail-famille? Quelle est l'attitude de leur entreprise à l'égard de l'avancement des femmes?

\section{Méthodologie}

Les verbatim des dix-huit entrevues ont été soumis à une analyse de contenu de type thématique et les données ont été organisées autour de deux grandes catégories: la perception qu'ont les femmes cadres du contexte organisationnel dans lequel elles travaillent et leur perception d'elle-même dans ce milieu. Ces données ont permis de développer une herméneutique de leur vécu quotidien et de cerner dans quelles mesures elles sont confrontées à des obstacles liés au genre, et si elles se voient comme «facteur de changement» pour l'avancement des femmes dans des entreprises traditionnellement masculines.

Afin de nous donner une marge de manoeuvre suffisante pour répondre aux situations multiples dans lesquelles se trouvent les femmes cadres des entreprises à l'étude, nous avons retenu la définition large d' «administratrice» développée par Harel-Giasson. Celle-ci présente les femmes cadres comme «des personnes qui ont des subordonnés sous leurs ordres et qui exercent une fonction-conseil de type professionnel au sein de la hiérarchie organisationnelle» (1981:61). 


\section{Les résultats}

\subsection{Perception du contexte organisationnel}

Pour mettre en contexte leur perception d'elles-mêmes, en tant que femmes cadres dans l'entreprise, nous avons voulu comprendre comment elles voient la structure d'ensemble de l'organisation où elles travaillent. Nous leur avons demandé de parler de cette question en fonction de trois aspects:

1) la dimension institutionnelle (mode de gestion, rapports administration-syndicat, notamment le climat des négociations des conventions collectives, et, globalement, le rapport aux règles);

2) la dimension culturelle (s'agit-il d'une culture de conflit ou de coopération et comment celle-ci se manifeste-t-elle?) et,

3) la dimension du fonctionnement organisationnel, qui permettait d'identifier leur satisfaction ou leur insatisfaction à l'égard de la façon dont les choses se passent, à l'intérieur, dans la gestion quotidienne (importance de la hiérarchie, possibilité de travail d'équipe, exclusions, etc.).

Retenons les résultats obtenus à partir de ce dernier aspect, qui sont particulièrement intéressants pour le sujet qui nous occupe puisqu'ils résument les éléments précédents et expriment de façon claire sous quelle tension continue se vivent les rapports quotidiens de travail.

D'un côté, les femmes se disent généralement satisfaites de l'ensemble de la structure organisationnelle de leur entreprise, relevant des aspects positifs en ce qui a trait à leur employeur (la Compagnie), à leur équipe de travail ou même, pour certaines, à leur supérieur. Pour donner quelques exemples: en ce qui concerne la Compagnie, quelques-unes invoquent ses valeurs de base, indiquant que tout se passe de façon «très professionnelle» $\left(\mathrm{R}_{15}\right)$, «business like» $\left(\mathrm{R}_{8}\right)$, que «the Company is looking for the best» $\left(\mathrm{R}_{11}\right.$; $\left.\mathrm{R}_{16}\right)$ ou " is supportive of education» $\left(\mathrm{R}_{7} ; \mathrm{R}_{14}\right)$. D'autres insistent sur la qualité de leur équipe: «fortunate to get a good crew» $\left(\mathrm{R}_{6}\right)$, «it's a team effort» $\left(\mathrm{R}_{8}\right)$, "a good team" $\left(\mathrm{R}_{6}\right)$, "good working relationship with employees» $\left(\mathrm{R}_{2}\right)$. D'autres encore feront des remarques sur 
"...le fait de travailler dans une culture masculine et le fait d'être en minorité sont des variables incontournables de la construction de leur réalité quotidienne. " l'organisation comme telle: «well structured» $\left(\mathrm{R}_{16}\right)$, «safety oriented» $\left(\mathrm{R}_{14}\right)$; sur le climat: «climate adequate to take decision» $\left(\mathrm{R}_{1}\right)$; ou sur leur patron: «respect and listen to your opinions» $\left(\mathrm{R}_{4}\right)$, «involvement of superintendant» $\left(\mathrm{R}_{5}\right)$. Toutes ces remarques font voir qu'elles ont une évaluation d'ensemble assez positive de leur milieu de travail, un «regard en extériorité» leur autorisant la distance nécessaire pour rationaliser leur vécu en fonction de valeurs fondamentales.

D'un autre côté, dès qu'elles entrent dans des aspects plus individuels et plus personnels, les obstacles, les insatisfactions se font souvent jour et on entrevoit, au travers de remarques diverses, à quel point le fait de travailler dans une culture masculine et le fait d'être en minorité sont des variables incontournables de la construction de leur réalité quotidienne. Ainsi, après avoir fait quelques commentaires généraux négatifs sur l'organisation «decision making vague» $\left(\mathrm{R}_{10}\right)$, «très peu de planification, d'encadrement» $\left(\mathrm{R}_{15}\right)$, «division does not have clear decision making» $\left(\mathrm{R}_{10}\right)$ - ou sur le formalisme des relations et des attitudes — «it's a real protocol (be careful what you say and what you do)" $\left(\mathrm{R}_{16}\right)$, «older people difficult time accepting changes» $\left(\mathrm{R}_{6} ; \mathrm{R}_{11}\right)$, «everybody has a boss》 $\left(\mathrm{R}_{11} ; \mathrm{R}_{4}\right)$ , inévitablement, les plus grandes frustrations viennent du fait de travailler dans un environnement dominé par les hommes:

«rarely women on the team» $\left(R_{3} ; R_{18}\right)$, «it's a male environment" $\left(R_{4}\right)$, "still some isolated areas (men have some restrictions)» $\left(R_{12}\right)$, «difficulties in dealing with some people in marketing (male populated)» $\left(R_{8}\right)$, «tough environment» $\left(R_{4}\right)$, «control environment» $\left(R_{7}\right)$, «inherited type of behaviour that's traditionnal to the Company" $\left(R_{17}\right)$, "Company very male dominated workplace» $\left(R_{7}\right)$, "none of the women at higher level have real power» $\left(R_{14}\right)$, «at the top of the pyramid you see less women» $\left(R_{1}\right)$.

Cette tension, cette obligation de vivre chaque jour avec la nécessité de se découper un espace dans un univers saturé au masculin, que l'on retrouvera encore plus explicitement exprimée lorsqu'elles parleront des rapports directs avec leurs collègues de travail, n'est pourtant ni aussi simple qu'il n'y parait, ni si facilement 
«... [les femmes] partagent toutes une ambition commune, une certaine détermination dans la poursuite de leur carrière. ") repérable, en ce qu'elle est construite sur un ensemble de contradictions, comme on peut le constater dans leurs différentes trajectoires.

\subsection{Perception de soi comme femme cadre dans l'entreprise}

On se rend compte assez rapidement, à écouter les participantes, que, bien qu'elles aient eu des trajectoires diversifiées, elles partagent toutes une ambition commune, une certaine détermination dans la poursuite de leur carrière. On réalise aussi que, bien que la plupart d'entre elles aient été encouragées dans leur milieu de travail à poursuivre cette carrière, elles sont confrontées, en tant que femmes, à des obstacles liés aux stéréotypes.

\section{- Trajectoires éclatées}

Les circonstances qui les ont menées à leur poste actuel sont diversifiées. Cela peut aller d'une embauche à travers une «firme de consultants», au fait que la compagnie «avait entendu parler d'elle», ou que, comme dans un des cas, la personne se soit organisée pour "créer elle-même son propre poste». Mais la situation la plus courante est que les femmes sont déjà en place et considérées qualifiées pour le poste qui s'ouvre: «direct supervisor knew my background», "approach by my boss», "promotion», "nomination», "recall back to the office». Dans un seul cas, la personne interviewée a signalé le fait qu'elle fut embauchée parce qu'il y avait un «bonus to the Company if hiring younger people».

\section{- Compétence et motivation}

À la question, pourquoi croyez-vous que vous avez obtenu le poste que vous détenez en ce moment, et quel en était l'intérêt pour vous, les réponses obtenues, comme on le constate ci-dessous, font clairement ressortir que l'on fait ici face à un échantillon de femmes déterminées, carriéristes, conscientes de leur potentiel, de leurs qualifications et, de plus, très ambitieuses. Voici quelques exemples, en ce qui a trait à leur perception de leurs qualifications et de leur compétence: 
"I was more qualified for the job» $\left(R_{14}\right)$, "I was the logical choice» $\left(R_{2}\right)$, "I was already doing the job» $\left(R_{8}\right)$, «I was familiar with the ins and outs (...) I took a training and the Company made commitment to hire me» $\left(R_{6}\right)$.

ou en ce qui concerne leur détermination pro-active:

«worked my way up through various level of the organizations» $\left(R_{2}\right)$; "pushed myself hard [...] took the initiative [...]. I took the chance» $\left(R_{5}\right)$; "ploughed my way in the Company for that position [...] you use your own initiative and competitiveness to make a place for yourself in the organization [...] I wanted to progress [...] I knew that there was something I could contribute» $\left(R_{17}\right)$; "I wanted a job with my qualifications $[. .$.$] I wanted to move [. .$.$] get some$ more opportunities» $\left(R_{1}\right)$; «je voulais développer mes aptitudes pour diriger et agir» $\left(R_{q}\right)$; "good for my career development to have the experience» $\left(R_{3}\right)$.

\section{- Appui des supérieurs}

Lorsqu'on leur demande si elles ont été encouragées à postuler, il n'y en a que trois qui diront que personne ne les a soutenues et deux qui signaleront que l'appui est venu de leur mari. Pour toutes les autres, ce sont des personnes (majoritairement des hommes) de leur milieu de travail. Onze des dix-huit femmes interviewées déclarent que c'est un supérieur (my boss: 6; the supervisor: 3; the manager: 2) qui les ont encouragées. Deux, seulement, parleront d'un ami ou d'un collègue.

Dans un monde qu'elles définissent elles-mêmes comme extrêmement compétitif et où la domination masculine s'exerce sur une base considérée comme habituelle et normative, n'est-il pas étonnant d'entendre que les supérieurs masculins encouragent les femmes à obtenir des postes où elles auront plus de responsabilités, plus de pouvoir? Mais qui sont ces patrons qui appuient l'avancement des femmes? 
«...plus le poste qu'elles occupent est élevé, dans la hiérarchie, plus il semble qu'elles sont en butte aux attitudes sexistes. "

\section{- Obstacles au quotidien}

\section{- Le sexisme ordinaire}

C'est dans l'analyse des manifestations tangibles du sexisme dans leur environnement de travail que se trouve la réponse à cette question. Ces manifestations sont de deux ordres:

1) l'attitude des collègues masculins à leur égard, en tant que femme, qui renvoie en fait, à tout ce qui touche globalement au rapport au genre et,

2) la résistance aux changements qu'elles veulent promouvoir, notamment en ce qui a trait à certains modes de gestion.

Alors même qu'elles se disent encouragées par leurs supérieurs dans l'avancement de leur carrière, elles déclarent aussi, dans le même temps, être en butte à l'attitude sexiste de leurs collègues de travail, quand ce n'est pas de leur supérieur immédiat. Et plus le poste qu'elles occupent est élevé, dans la hiérarchie, plus il semble qu'elles sont en butte aux attitudes sexistes. En fait, on réalise que plusieurs de celles qui ont déclaré avoir été soutenues par un patron faisaient référence à une personne à qui elles ne répondent pas directement dans leur travail quotidien, mais plutôt à un supérieur plus important dans la hiérarchie. Parce que, dans l'univers immédiat des rapports de pouvoir, il s'agit d'une autre réalité. En effet, se vivent au jour le jour des difficultés de relations entre collègues ou entre patrons/employées, exacerbées par le sexisme ambiant qui sexualise les relations de telle sorte qu'elles sont obligatoirement interprétées, à prime abord, comme des rapports hommes/femmes; ainsi que l'expriment certaines d'entre elles:

"My worst relationship is with my direct boss [...] I don't get any support from my boss» $\left(R_{10}\right)$; «male superintendent becoming problematic at work [...] they didn't want me as part of their team, so they hired someone with absolutely no experience» $\left(R_{13}\right)$; «still remarks made from time to time on women" $\left(R_{12}\right)$.

Difficultés où s'expriment le manque de confiance de la part de leur environnement immédiat, parce qu'elles sont des femmes: 
"...les hommes ne veulent pas savoir ce que pensent les femmes qui travaillent avec eux..." «men don't think I can do it on my own» $\left(R_{18}\right)$; «new general foreman thinks I have no good ideas, ideas have to come from him» $\left(R_{8}\right)$; "difficult breaking cultural barrier to get ideas accepted» $\left(R_{1}\right)$.

et leur sentiment d'exclusion:

"Never know where I fit into the group» $\left(R_{18}\right)$; «no way they want to work with women» $\left(R_{12}\right)$; «it was the old boy's club [...] you have to be careful because men are very cryptic, I find, and they certainly don't take well to what they feel is excitement on your part" $\left(R_{1}\right)$; «unfair that men are sent to the plant and I was left behind because I was a female» $\left(R_{12}\right)$.

Elles ne sont pas, semble-t-il, les bienvenues dans certains milieux, et certaines jalousies et conflits interpersonnels se développent: "a lot perceive I haven't earned the position» $\left(\mathrm{R}_{17}\right)$; «we have run ins with the guys» $\left(\mathrm{R}_{18}\right)$; «I have a lot of adversary» $\left(\mathrm{R}_{16}\right)$. En fait, selon le résumé qu'en fait l'une d'entre elles, les hommes ne veulent pas savoir ce que pensent les femmes qui travaillent avec eux:

Jusqu'à quel point on est prêt à écouter, et je pense que c'est le mot clé, "écouter», écouter ce que madame a à dire au même titre que ce que monsieur a à dire. C'est dur comme femme, c'est lui qu'on va écouter [...] c'est une question hiérarchique encore. Ça dépend de qui vient de parler $\left(R_{15}\right)$.

Mais, il va sans dire que, bien que cela soit répandu, toutes ne sont pas aux prises avec une telle discrimination. Certaines se sentent appuyées dans leurs décisions en tant que gestionnaires, quatre ont signifié avoir de bonnes relations avec leur patron et cinq disent qu'elles reçoivent du «feedback» positif (mais pas nécessairement de leur patron direct). 


\section{- Un autre enjeu: instauration de nouvelles formes de rapports d'autorité}

Un des blocages que les femmes semblent avoir dans l'exercice de leurs fonctions est que certaines d'entre elles tentent d'instaurer des pratiques plus participatives au niveau de la prise de décision et de la répartition des tâches. Elles tentent de transformer le mode de gestion traditionnel dans leurs équipes de travail: «we try to work together as a team» $\left(\mathrm{R}_{4}\right)$, "we try to reach consensus within the group» $\left(\mathrm{R}_{2}\right)$, «constant explanations, discussions» $\left(\mathrm{R}_{1}\right)$, «consult with each othen $\left(\mathrm{R}_{10}\right)$, «discuss with the team» $\left(\mathrm{R}_{3}\right)$, «we visit the workplace» $\left(\mathrm{R}_{2}\right)$, «and talk to the workers» $\left(\mathrm{R}_{4}\right)$. Ce faisant, elles semblent rencontrer beaucoup de résistance. L'une dira: «when you get in the group and you start raising anxiety levels and wanting different things and wanting them to work as a team where they have been allowed to work independently, it does create discomfort» $\left(\mathrm{R}_{7}\right)$.

Les hommes ne font pas confiance aux femmes en tant que collègues. Ce monde d'hommes ne leur permet pas si facilement d'apporter quelque chose de différent à leur mode de fonctionnement, notamment l'humanisation des pratiques de gestion, la collégialité participative ou encore la recherche du consensus en lieu et place d'une imposition de l'autorité dans les décisions, comme le démontrent certains commentaires entendus. En se sens, leurs façons de faire, lorsqu'elles sont différentes de celles de la culture masculine, peuvent aussi être un obstacle à leur avancement.

\section{- La conciliation travail-famille: un sujet tabou?}

Aux termes des obstacles à leur vie de femme professionnelle, il en est un, pourtant que l'on se serait attendu à trouver: le handicap que pourrait constituer le fait d'avoir une famille, ou, à tout le moins, plusieurs enfants. Peu d'entre elles en ont fait explicitement mention. Cela est d'autant plus étonnant que les deux tiers d'entre elles ont des responsabilités familiales: «for not wanting a higher position in the Company», «family demands» $\left(\mathrm{R}_{14}\right)$, «time» $\left(\mathrm{R}_{2}\right)$. Pourtant, dans l'autre sous échantillon de la même enquête, celui des cadres des Caisses populaires, cette thématique avait été abondamment soulevée par les femmes interviewées. 
«...les femmes travaillant dans les secteurs non traditionnels, doivent encore, si elles veulent faire carrière, effectuer un choix entre avoir des enfants ou développer une carrière. "
Peut-on penser qu'au sein d'un milieu typiquement masculin, c'est une question qui est encore de l'ordre du tabou, de l'impensé? Que ces femmes ne veulent surtout pas faire intervenir leur vie privée dans leur milieu professionnel? Ont-elles à ce point intégré la norme de la division public/privé qu'elles ne veulent présenter d'elles-mêmes que leur identité professionnelle, par peur de ne pas être considérées au même titre qu'un collègue masculin? À l'appui de cette hypothèse, signalons une recherche récente où l'auteur démontre que les femmes travaillant dans les secteurs non traditionnels, doivent encore, si elles veulent faire carrière, effectuer un choix entre avoir des enfants ou développer une carrière (Ranson 1998).

Une chose est certaine, on ne peut croire que la question de la conciliation travail-famille peut se résoudre aussi facilement (Descarries et Corbeil 1995; 1996), même si l'on sait que plus les femmes sont éduquées, plus elles ont accès à des services permettant une meilleure gestion de cette articulation.

\subsection{Et les autres femmes de l'entreprise?}

Bien qu'elles identifient assez clairement le sexisme auquel elles doivent faire face journellement, la plupart d'entre elles considèrent que la Compagnie est assez favorable à l'avancement des femmes. Comme le résume l'une d'elles «the institution allows women in decision making roles and is open minded and fain $\left(\mathrm{R}_{6}\right)$. Six des femmes iront jusqu'à affirmer que «it's not a problem to move up in the organization» $\left(\mathrm{R}_{2}\right)$. Par contre, d'autres soulignent que la compagnie «is a very male dominated workplace [...] we have a long way to go» $\left(\mathrm{R}_{7}\right)$, que les hommes «don't go out of their way to help women» $\left(\mathrm{R}_{14}\right)$ et que, bien qu'il y ait eu des ouvertures pour les femmes, «none has been promoted» $\left(\mathrm{R}_{16}\right)$. L'une affirmera même que «as you get to the top of the pyramid, I mean, you get a hell of a lot smaller [...] it's harder and harder for women to get up and up the ladden $\left(\mathrm{R}_{18}\right)$.

Dans ce tissu d'ambiguïtés, qui est à l'image des contradictions dans lesquelles elles se débattent au quotidien, un élément est apparu révélateur. Quelques-unes ont indiqué que, dans 
l'entreprise, il y a une certaine entraide des femmes: «women within the organization are helping people, like helping other women» $\left(\mathrm{R}_{3}\right)$. Parlons-nous ici du développement timide d'une certaine solidarité féminine?

\section{Discussion}

"...elles doivent

même être plus

compétentes, souvent, que leurs collègues masculins pour obtenir des postes équivalents. "
Que retient-on des témoignages de ces femmes cadres? Quelle perception ont-elles d'elles-mêmes, de leur milieu de travail et de leur rôle en tant que facteur de changement dans l'entreprise, ou par rapport à l'avancée des femmes?

Dans l'ensemble, on a pu voir que toutes les femmes interviewées sont déterminées à monter les échelons de la hiérarchie, qu'elles ont le sentiment réel de poursuivre une carrière, ce qui signifie progression, apprentissages nouveaux et avancement, et ce malgré le fait qu'elles aient, la plupart d'entre elles, une famille. Elles ont toutes signalé qu'elles étaient très qualifiées pour occuper leur poste, dans certains cas «la plus qualifiée», et qu'elles avaient la capacité de gérer et d'assumer des responsabilités. Certaines ont aussi exprimé l'idée répandue selon laquelle elles doivent même être plus compétentes, souvent, que leurs collègues masculins pour obtenir des postes équivalents. Par ailleurs, elles semblent satisfaites de leur situation, mais avec l'idée que celle-ci doit évoluer. Pour elles, et comme l'avaient déjà fait voir Harel-Giasson et Marchis-Mouren (1988), ce qui contribue à leur avancement dans l'entreprise est d'abord et avant tout leur compétence en matière de direction, mais aussi leur conviction en cette compétence.Et, en grande partie, les comportements institutionnels leur donne raison. Certes, elles bénéficient de certains appuis dans l'entreprise. Ceci, d'ailleurs, confirme ce qui a été avancé par Poirier, qui a démontré que celles qui réussissent à accéder à des postes cadres ont généralement bénéficié de l'aide de parrains, haut placés dans la hiérarchie, leur permettant de «développer leur technique, de démontrer beaucoup de professionnalisme et aussi de produire des résultats visibles» (1992:17).

Mais pourquoi obtiendraient-elles un tel appui de leurs supérieurs plus éloignés si ce n'est qu'effectivement leurs qualifications, leur savoir-faire, sont nécessaires, utiles à la compagnie? 
C'est ici où se font jour les contradictions du système, où la logique économique prend le pas sur la symbolique patriarcale des stéréotypes sexués. Lorsque le motif constitue un enjeu supérieur, du point de vue de l'organisation, on accepte de reconnaître la valeur des femmes. Mais cette reconnaissance même, comme on l'a vu, bien qu'elle soit un atout pour les femmes en place, ne parvient pas à transformer l'économie des rapports sociaux de sexe dans la gestion quotidienne des relations de travail. Au contraire, elle, demeure profondément sexiste au sein de plusieurs milieux où les femmes évoluent, surtout lorsqu'elles occupent un poste de responsabilité, parce que la mentalité qui la soustend demeure, elle, profondément ancrée chez les travailleurs. Ce sont principalement les collègues masculins, ceux de même niveau hiérarchique, beaucoup plus souvent que les employés de leurs équipes, ainsi que certains de leurs supérieurs directs, qui posent problèmes aux femmes interviewées. Ces problèmes semblent, d'ailleurs, être de deux ordres: la confiance et l'inclusion.

Il est apparu clairement que, dans une certaine proportion, leur environnement masculin ne leur fait pas confiance. Cela s'exprime de diverses façons: soit on ne croit pas que la femme arrivera à faire le travail seule; soit on la protège de certains environnements en lui interdisant d'y aller; soit on refuse tout simplement de l'écouter exprimer ses idées, comme si elle ne savait pas penser... tous des comportements typiques d'une attitude sexiste. Landry (1990:132) avait déjà identifié ce manque de confiance des hommes en leurs collègues féminines. Ils semblent vivre là un sentiment d'insécurité profonde (une perte de contrôle?) qui s'exprime dans leur incapacité de reconnaître la compétence de leurs collègues femmes comme si, subitement, les règles de la compétition et de la concurrence étaient transformées à leur désavantage.

On pourrait voir comme une autre conséquence de ce sentiment, le mouvement de repli sur soi, le renforcement des barrières d'inclusion du groupe des hommes au détriment des nouvelles venues tel que l'avait signalé Kanter (1977) dans sa typologie des rapports de pouvoir. Plusieurs des femmes ont fait état de ces comportements masculins: ils refusent que la femme cadre fasse 
"C'est cette impossibilité de forcer la porte des réseaux informels qui constitue probablement le plus grand obstacle auquel sont confrontées les femmes, les excluant des systèmes d'ouvertures ou de possibilités. " partie de l'équipe; ils la rabrouent lorsqu'elle manifeste son enthousiasme pour un projet; ils la font se sentir de trop dans diverses situations.

On voit bien ici, dans ces comportements d'exclusion, dans ce manque de confiance, la construction des barrières liées au genre, celle du "plafond de verre», et à la structure sexiste traditionnelle du marché du travail, qui se déploient d'autant mieux qu'on est en présence d'entreprises où les postes de direction ont été longtemps - et sont encore — l'apanage des hommes (Colwill 1987; Lasvergnas 1994). C'est cette impossibilité de forcer la porte des réseaux informels qui constitue probablement le plus grand obstacle auquel sont confrontées les femmes, les excluant des systèmes d'ouvertures ou de possibilités (opportunités), parce que, dans une organisation, les réseaux d'information informels sont au moins tout aussi essentiels à la compréhension des enjeux, à la connaissance des paramètres internes, à la perception des canaux sous-jacents du pouvoir, que les réseaux officiels, visibles (Crozier 1977).

En ce qui a trait à la culture organisationnelle comme telle, là aussi, on peut constater la difficulté, pour les femmes cadres, d'être en phase avec le milieu. Plusieurs des femmes ont mentionné le fait qu'elles tentent d'instaurer de nouvelles pratiques de gestion, basées plus sur une certaine collégialité participative, une recherche du consensus, que sur une imposition de l'autorité dans les décisions. Mais elles ne semblent guère être appuyées en ce sens. Encore ici, on parle de conflits avec les collègues, d'incompréhensions: l'innovation des pratiques, dans l'entreprise, ne doit pas venir d'elles, sinon il semble y avoir surenchère au niveau de l'obligation d'adaptation qu'elles exigent du milieu. Mais, peut-on se demander, comment seraient interprétées ces initiatives si elles n'étaient pas le fait des femmes cadres, mais le fait de leurs collègues masculins? Dans toutes ces situations où elles sont confrontées à des refus, à des fins de non recevoir, à une nécessité, souvent, de se plier à la volonté de la majorité, on peut constater que les stratégies qu'elles déploieront le plus fréquemment, soit développer une résistance subtile ou dépolitiser les enjeux, manifestent bien la teneur de la trame de fond sur laquelle se construisent leurs rapports de travail. Ainsi que le dira cette participante, parlant de 
"...il semble admis que plus une femme a un poste élevé, plus elle est confrontée à des conflits liés à la culture organisationnelle. » ses collègues masculins: «It's important that you ask them their opinion, and that you take that into consideration, or at least acknowledge that you considered it» $\left(\mathrm{R}_{18}\right)$. La question que l'on peut se poser à ce propos, c'est inévitablement: lui rendent-t-ils la pareille? Mais quelle que soit l'analyse que l'on pourrait en faire, ne peut-on penser que les notions de «marge de manoeuvre» ou de «liberté de l'acteur», telles que les travaux en sociologie des organisations les ont développés, s'appliquent peu aux situations des femmes inscrites dans de tels milieux? La «liberté de l'acteur» semble nettement plus «mince» lorsque, non seulement il faut «performer» plus que les collègues masculins pour réussir et être acceptée, mais qu'en plus, il faut le faire sans trop choquer, en contournant les stéréotypes en quelque sorte.

Il est un autre élément que la présente analyse permet de constater, c'est que, pour rendre compte des stratégies d'action des femmes en position de pouvoir dans des entreprises typiquement masculines, il faut raffiner nos instruments d'analyse. On ne peut plus penser que leurs stratégies de réponses peuvent être appréhendées de façon globale, pour toutes les femmes d'une entreprise, ou en fonction de sa culture organisationnelle globale, comme le modèle de DesRosiers (1993) les a construites. C'est en fonction des catégories de postes occupés qu'il faudrait désormais le faire, puisqu'il semble admis que plus une femme a un poste élevé, plus elle est confrontée à des conflits liés à la culture organisationnelle. Il y aurait aussi lieu de développer une lecture plus sensible de la sexualisation de chaque environnement immédiat relativement aux rapports hommes/femmes: les femmes de notre échantillon ne sont pas toutes, en effet, soumises aux mêmes manifestations du sexisme.

Finalement, se voient-elles agentes de changement en ce qui concerne l'avancée des femmes dans les postes traditionnellement masculins? Elles abordent peu cette question, ne se définissent pas comme symbole social ou comme modèle. Simplement, elles sont conscientes que tant qu'il n'y aura pas plus de femmes aux plus importants postes de pouvoir («none of the women at high level have real powen), les choses auront peu de chance de se transformer. 
«Du seul fait de leur présence dans ces postes, elles agissent sur les symboliques sociales dans le sens d'une transformation des diktats de l'idéologie patriarcale."
Toutefois, du point de vue de l'analyse sociologique, on peut penser que, oui, elles sont facteurs de changement. Du seul fait de leur présence dans ces postes, elles agissent sur les symboliques sociales dans le sens d'une transformation des diktats de l'idéologie patriarcale.

En outre, certaines d'entre elles ont indiqué qu'elles «encourageaient et aidaient leurs collègues femmes», ce qui inviterait à reconnaître le début d'une certaine «solidarité féminine», une action concrète des femmes entre elles dans l'entreprise, mais qui aurait peu l'occasion de s'exprimer, parce que les femmes, selon leurs dires, sont trop peu nombreuses et travaillent souvent éloignées les unes des autres, dans différents secteurs. Si le nombre de femmes augmentait dans l'entreprise, et particulièrement dans les postes de responsabilité, pourrait-on s'attendre à ce que cette forme inchoative de "solidarité féminine», ou à tout le moins le développement de «réseaux informels version femme» devienne propice à la transformation de la culture organisationnelle, à l'amélioration des modes de gestion traditionnels, particulièrement dans les milieux où ils sont de type rigide, non flexible? Seule une étude à ce sujet portant sur des entreprises où les femmes seraient en nombre relativement important dans les postes de pouvoir pourrait le dire.

Quoiqu'il en soit, cette nouvelle solidarité des femmes en situation de gestion, si elle se développait, même de façon timide, pourrait sans doute être, comme le signale Guay «un des maillons importants de l'avenir des femmes dans des postes d'influence» (1992: 64).

\section{Conclusion}

L'analyse a démontré qu'il y a un ensemble de facteurs qui concourent à l'accès des femmes à des postes de responsabilité et à leur avancement dans une entreprise typiquement masculine.Autant leur profil de femmes éduquées que leur détermination de femmes 
"Sans une transformation réelle des stéréotypes sexistes, sans une nette percée en faveur de l'égalité dans toutes les couches de la société, les milieux de travail traditionnellement masculins resteront en grande partie ce qu'ils sont..." ambitieuses et compétentes - qui en font des individus très compétitifs dans la recherche d'emploi ou de promotion -, que la transformation des valeurs sociétales - qui oblige les entreprises à ouvrir leurs portes aux femmes et à en nommer quelques-unes à des postes de cadre supérieur —, sont au nombre des éléments à retenir.

Cependant ces éléments, même s'ils sont essentiels à l'avancement des femmes, ne sont pas, en soi, suffisants pour le garantir. Ni l'éducation supérieure des femmes, ni leur compétence, ni leur conviction même en cette compétence, ne peuvent faire en sorte, comme l'ont démontré les vécus de femmes présentés ici, que se transforme la culture traditionnelle de l'entreprise et que se développe la volonté politique de faire en sorte que les femmes atteignent les plus hautes sphères de la hiérarchie. Sans une transformation réelle des stéréotypes sexistes, sans une nette percée en faveur de l'égalité dans toutes les couches de la société, les milieux de travail traditionnellement masculins resteront en grande partie ce qu'ils sont: des environnements où l'on manifeste peu de confiance aux femmes, où la valorisation de leur compétence se fait au prix de leur exclusion des réseaux de collégialité.

De même, sans une augmentation substantielle du nombre de femmes dans ces entreprises, il y a peu d'espoir d'amélioration tangible:le poids des nombres, tel que l'ont indiqué Kanter (1977) et DesRosiers (1993) est un poids réel, toutes les participantes à l'enquête en ont fait état. Mais, ont-elles dit, surtout, ce qui importe est une augmentation des femmes dans les postes de responsabilité et dans les hautes sphères de la hiérarchie. Car ce n'est pas tant le pouvoir des nombres, qui, somme toute, fait la différence, mais bien le nombre au pouvoir.

Peut-on alors espérer, puisque le nombre d'ingénieures et le nombre de diplômées en sciences est croissant dans la société canadienne, que l'on assistera éventuellement à une véritable transformation des rapports entre les sexes dans les milieux traditionnellement masculins? Une percée qui permettrait d'aller au-delà de cette pratique sexiste de la culture organisationnelle qui ne veut voir dans les relations des hommes et des femmes dans les milieux de travail que des rapports sexués, que de 
l'interaction toujours déjà sexualisée? Une transformation qui ferait éclater la logique historique sexiste des rapports de travail?

\section{Bibliographie}

ARMSTRONG, Pat et Hugh ARMSTRONG (1994) (1978). The Double Ghetto, 3rd ed., Toronto, McClelland and Stewart.

ARMSTRONG, Pat et Hugh ARMSTRONG (1990). Theorizing Women's Work, Toronto, Garamond Press.

BARRÈRE-MAURISSON, Marie-Agnès (1992). La division familiale du travail: la vie en double, Paris, Presses Universitaires de France.

BERNIER, Christiane et Simon LAFLAMME (2001). «S'éduquer et sortir des ghettos roses: des stratégies féministes efficaces?», dans Actes du colloque de l'ICREF/CRIAW, Gabrielle Lavigne, Tricia Burke et Manon Lemonde, éd., "Les définitions féministes des modes de vie sains et des sociétés sensibles à l'être humain", Sudbury, The Scrivener Press, 135-154.

BERNIER, Christiane et Simon LAFLAMME (2000). «L'équité salariale au Canada: un progrès inégal», Géographie, Économie et Société, vol. 2, no 1,149-178.

CODERRE, Cécile, Ann DENIS et Caroline ANDREW (1999). Femmes de carrière, carrière de femmes: étude des trajectoires familiales, scolaires et professionnelles des gestionnaires québécoises et ontariennes, Ottawa, Les Presses de l'Université d'Ottawa, Études des femmes.

COLWILL, Nina (1987). «Men and women in organizations: roles and status, stereotypes and power», dans Karen Shallcross Koziara, Michael H. Moskow et Lucretia Dewey Tanner, éd. Working Women: past, present, future, Washington, The Bureau of National Affairs, 97-117.

COMMISSION DES SCIENCES ET DE LA TECHNOLOGIE AU SERVICE DU DÉVELOPPEMENT (1996). L'autre développement: l'égalité des sexes dans la science et la technologie, Ottawa, Centre de Recherches pour le Développement International.

CONSEIL CONSULTATIF NATIONAL DES SCIENCES ET TECHNOLOGIES (1993). Les femmes, un atout dans les métiers, la technologie, les sciences et le génie, Ottawa, Rapport du Conseil consultatif national des sciences et de la technologie.

CROZIER, Michel et Erhard FRIEDBERG (1977). L'acteur et le système, Paris, Éditions du Seuil.

DAVID, Hélène (1986). Femmes et emploi: le défi de l'égalité, Sillery, Presses de l'Université du Québec.

DAVIS, Fran et Arlene STEIGER (1995). «Les femmes qui entrent dans la culture scientifique», dans Association pour la Recherche au Collégial: Actes du 7e colloque de l'A.R.C. - Sciences, technologie et communication - Au collégial, un monde branché, Montréal, Association pour la recherche au collégial, http://infopuq.uquebec.ca.

DELPHY, Christine (1998). De l'exploitation familiale au concept de genre, Montréal, Université du Québec à Montréal, Thèse présentée comme exigence partielle du doctorat en sociologie.

DELPHY, Christine (1970). «L'ennemi principal», Partisans, vol. 54-55, 157-172.

DESCARRIES, Francine et Christine CORBEIL (1996). «Concilier travail et famille», dans Huguette Dagenais et Micheline Beauregard, éd., Science, conscience et action, Montréal, Éditions du remueménage. 
DESCARRIES, Francine et Christine CORBEIL (1995). « Ré/conciliation famille-travail: les enjeux de la recherche ", Cahiers Réseau de recherches féministes, Université du Québec.

DESCARRIES, Francine et Christine CORBEIL (1995). Famille et travail: double statut...double enjeu pour les mères en emploi, Montréal, Université du Québec à Montréal.

DESROSIERS, Micheline (1993). «Stratégies d'influence et contexte organisationnel», dans Lou Hammond Ketilson, éd. Femmes et gestion, Saskatchewan, Université de Saskatchewan, 52-59.

DEVREUX,Anne-Marie (1985). «De la construction féminine aux rapports sociaux de sexe: repères pour une évolution de la définition sociologique des catégories de sexe», BIEF, vol. 16, 13-23.

FIELD, Debbie et Joanne SANTUCCI (1983). «Working Steel» dans Jennifer Penny, Hard Earned Wages, Women Fighting for Better Work, Toronto, The Women's Press.

GUAY, Marie-Michèle (1992). «Carrières de femmes cadres au mitan de la vie», Gestion, vol. 17, février, 60-67.

GUILLAUMIN, Colette (1978). «Pratique du pouvoir et idée de Nature: (1) L'appropriation des femmes», Questions féministes, vol. 2, février, 5-30.

GUNDERSON, Morley et Craig RIDDEL (1988). Labour Market Economics: Theory, Evidence and Policy in Canada, Toronto, McGraw-Hill Ryerson.

HAREL-GIASSON, Francine (1981). Perception et actualisation des facteurs de promotion chez les femmes cadres de grandes entreprises québécoises francophones du secteur privé, Montréal, Université du Québec à Montréal.

HAREL-GIASSON, Francine et Marie-Françoise MARCHIS-MOUREN (1988). «Que nous ont appris quinze ans de fréquentation de femmes cadres», dans Francine Harel-Giasson et Jeannine Robichaud, éd., Tout savoir sur les femmes cadres d'ici, Montréal, H.E.C.

KANTER, Moss Rosabeth (1977). Men and women of the corporation, New York, Basic Books.

KECK, Jennifer et Mary POWELL (1999). «Women into Mining Jobs at Inco: Challenging the Gender Division of Labour», dans 7th International Interdisciplinary Congress on Women, June 21, Generations: Women's Worlds 99, Tromso, Norway.

KEMPENEERS, Marianne (1992). Le travail au féminin: analyse démographique de la discontinuité professionnelle des femmes au Canada, Montréal, Presses de l’Université de Montréal.

LANDRY, Simone (1990). «Les femmes et la dynamique du pouvoir dans les groupes restreints», dans Roger Tessier et Yvan Tellier, éd. Pouvoirs et cultures organisationnelles, Québec, Presses de l'Université du Québec.

LASVER GNAS, Isabelle (1994). «La sexuation de la science: une question purement imaginaire?», Cahiers de recherche sociologique, vol. 3, 57-77.

LAUZON, Léo-Paul (1995). Place des femmes à titre d'administratrices et d'officiers dans les entreprises canadiennes, Québec, Presses de l'Université du Québec.

LEMIRE, Louise et Anik DES MARAIS (1998). «Femme et carrière - évolution, contrainte et gestion", colloque sur Enjeux et défis de la carrière des femmes dans l'administration publique, Beyrouth, vol. 2, no 3 octobre.

LUXTON, Meg et Ester REITER (1997). «Double, double, toil and trouble: Canadian women's experience of work and family», dans Patricia Evans et Gerda Wekerle, éd., Remaking the Welfare State: Women and the Canadian Experience, Toronto, University of Toronto Press. 
MATHIEU, Nicole Claude (1989). «Identité sexuelle/sexuée/de sexe? Trois modes de conceptualisation du rapport entre sexe et genre», dans Anne-Marie Daune-Richard et al., éd., Catégorisation de sexe et constructions scientifiques, Aix-en Provence, CÉFUP, 109-148.

MCFARLANE, Seth, Roderic BEAUJOT et Tony HADDAD (2000). «Time constraints an relative Resources as Determinants of the Sexual Division of Domestic Work», Canadian Journal of Sociology, vol. 25, no 1, 61-82.

NAFF, Katherine (1994). «Through the glass ceiling: Prospects for the advancement of women in the Federal civil service», Public Administration Review, Novembre/Décembre, vol. 54, no 6, 507514.

POIRIER, Michèle (1992). «Les entreprises et la carrière des femmes», Gestion, vol. 17, février, 6876.

RANSON, Gillian (1998). "Education, work and family decision making: finding the right time to have a baby", The Canadian Review of Sociology and Anthropology, vol. 35, no 4, 517-533.

RESKIN, Barbara (1984). Sex Segregation in the Workplace:Trends, Explanations, Remedies, Washington, DC, National Academy Press.

SHELTON, B.A. (1992). Women, Men and Time: Gender differences in Paid Work, Housework and Leisure, Westport, Greenwood.

STATISTIQUE CANADA (1995). Travail non rémunéré des ménages: mesure et évaluation, no 13-603F au catalogue, no 3.

STATISTIQUE CANADA et CONDITION FÉMININE CANADA (1997). Indicateurs économiques de l'égalité entre les sexes, Rapport présenté aux Ministres fédérale, provinciaux et territoriaux responsables de la condition féminine, no SW21-17/1997F au catalogue.

VANDELAC, Louise (1985). Du travail et de l'amour: les dessous de la production domestique, Montréal, Saint-Martin.

\section{Notes}

1. Les données présentées ici ont été proposées pour publication en anglais dans Canadian Women in Science and Engineering: Historical and Contemporary Perspectives, 2002.

2. Ceci comprend les données de toutes les catégories confondues: foresterie, architecture, génie civil, chimique, électrique, mécanique et autres. Source: 2000 Status of Women Supplement. CAUT/ ACPPU Journal, automne 2000, p. 5. Données construites à partir de Statistics Canada, Postsecondary Education Section. Unpublished data.

3. Pour ne donner qu'un exemple: en 1998, Inco Ltd, le plus grand producteur de nickel au Canada (et au monde) embauchait moins de 50 femmes comme travailleuses dans des postes traditionnellement masculins sur 4200 employés (Keck et Powell, 1999: 26), même si à une époque plus prospère, il y en a déjà eu jusqu'à une centaine.

4. Selon une étude de Léo-Paul Lauzon (1995) menée en 1992 auprès de 307 entreprises en Ontario, 112 (soit 36,5\%), n'avaient aucune femme à titre d'administratrice, c'est-à-dire de femmes cadres qui ont des subordonnés sous leurs ordres et qui exercent une fonction-conseil de type professionnel.

5. Les données de cette enquête ont été collectées dans le cadre d'un projet de thèse de M.A. en sociologie. 
6. Les répondantes ont été codifiées comme suit: de $\mathrm{R}_{1}$ à $\mathrm{R}_{18}$ et les enregistrements ont été faits majoritairement en anglais.

7. Il est intéressant de noter que 10 des 18 femmes interviewées ont deux enfants ou plus.

8. Il semble cependant que l'Union européenne a touché cette question récemment, Voir, $\mathrm{La}$ stratégie-cadre communautaire en matière d'égalité entre les femmes et les hommes (2001-2005), http:// europa.eu.int/comm/employment_social/equ_opp/strategy_fr.html 